\title{
CARCINOMA INFILTRANTE DE MAMA
}

Consideraremos en este capítulo el abordaje de los verdaderos carcinomas mamarios invasivos. Se orientarán los protocolos hacia el manejo del Carcinoma Ductal por ser el más común, aunque el enfoque en general no cambia demasiado en relación con las demás variantes histológicas, de las cuales describiremos las más frecuentes a continuación

\section{Carcinoma Ductal Infiltrante}

Es el tipo más frecuente; constituye del 65 al 80\% de todos los tumores mamarios. De acuerdo con la definición de la OMS se clasifican como ductales invasores todas aquellas lesiones que no puedan ser clasificadas en ninguna de las otras categorías.

Se incluyen en este grupo lesiones como carcinoma ductal con fibrosis productiva, carcinoma escirroso y carcinoma simple. También comprende tumores que exhiben parcialmente una o más características de otros tipos específicos de carcinoma (que generalmente son de mejor pronóstico) pero que no llegan a ser puros. De acuerdo con la literatura cerca del $30 \%$ de los carcinomas invasivos clasificados como ductales tienen una o más combinaciones y un poco más de la mitad de este grupo muestran un componente del tipo histológico tubular. También se incluyen en este grupo los casos de carcinoma ductal invasivo con enfermedad de Paget asociada.

La presentación clínica poco difiere de los demás carcinomas y es más frecuente entre los 45 y los 50 anos.

Por ser el carcinoma mamario una entidad extremadamente heterogénea desde el punto de vista clínico y patológico, es muy difícil predecir su comportamiento y la sobrevida debido a la amplia variedad de grados histológicos y la diferencia en respuesta de cada paciente. Los factores pronóstico mencionados en otra sección pueden orientarnos en este aspecto, sin embargo el mayor impacto en la evolución y el pronóstico lo constituye el tratamiento primario.

\section{Carcinoma Tubular}

Es una lesión conocida hace más de 100 anos, compuesta por elementos neoplásicos que recuerdan las estructuras ductales normales de la mama. Como puede encontrarse asociado a carcinoma ductal invasivo, para calificarlo como tubular se requiere que al menos el $75 \%$ del tumor sea tubular. En la forma pura no constituye más del $2 \%$ de los carcinomas mamarios. Usualmente son tumores pequenos y algunos autores lo consideran como un estado en la evolución de un gran número de tumores invasivos mamarios. Presentan metástasis axilares del 0 al $25 \%$. La forma pura usualmente es de buen pronóstico.

\section{Carcinoma Medular}

Este tipo de tumor, descrito desde la década del cuarenta, representa alrededor del $7 \%$ los carcinomas mamarios y se presenta en mujeres más jóvenes que los demás tipos histológicos (entre el 40 y el $66 \%$ son menores de 50 anos). Se dividen de acuerdo al patrón histológico en típicos y atípicos. Usualmente son de gran tamano, tienen tendencia a la degeneración quística y una baja frecuencia de metástasis axilares. El $82 \%$ de las pacientes con medulares típicos sobreviven 5 anos después del tratamiento quirúrgico y en presencia de metástasis axilares la sobrevida es del 50\%. Cuando el patrón histológico es atípico el pronóstico no es tan bueno, semejándose al del ductal infiltrante.

\section{Carcinoma Mucinoso}

Conforma del 1 al 2\% de los carcinomas mamarios. Debe distinguirse la forma pura de aquellos que contienen mucosa y elementos de carcinoma mucinoso sin llegar a ser puros. Es de crecimiento lento, tiene tendencia a hacer metástasis tardías en el curso de la enfermedad y normalmente es de buen pronóstico. Se presenta con mayor frecuencia en mujeres ancianas. Puede encontrarse asociado con el comedocarcinoma. El diagnóstico histopatológico diferencial incluye ciertas formas de fibroadenoma y mucoceles que simulan tumor.

En estudios comparativos con el carcinoma ductal invasor se ha encontrado una más baja frecuencia de metástasis, axilares y menor número de muertes. Se han descrito recurrencias sistémicas luego de 10 anos de tto inicial y una inusual pero fatal complicación es la embolía mucinosa, la cual puede producir un infarto cerebral.

\section{Carcinoma Lobulillar Infiltrante}

Su frecuencia varía del 3 al $15 \%$ en las diferentes series. Tiende a presentarse más en mujeres ancianas. Se ha sugerido que pueden originarse en un carcinoma lobulillar in situ y se presenta tumor en el seno contralateral entre el 6 y $28 \%$ de los casos, razón por la cual es muy importante realizar diagnóstico diferencial con el $\mathrm{Ca}$ ductal infiltrante. Su aspecto clínico es variable, desde masas de grandes proporciones hasta tumores inaparentes que se manifiestan como induraciones mal definidas sin masa dominante. Tiene tendencia a presentar metástasis a $\mathrm{SNC}$, tracto digestivo, ovarios y útero. El $50 \%$ de las pacientes que presentan metástasis axilares fallecen. Los tumores no detectados por mamografía frecuentemente pertenecen a esta categoría. La sobrevida a 5 y 10 anos en las pacientes con ganglios negativos es del 86 y $74 \%$ respectivamente. El factor pronóstico más importante está dado por el tamano del tumor y la presencia de ganglios comprometidos.

\section{Carcinoma Papilar}

La Clasificación Histológica de Tumores de Mama de la OMS lo define como «un carcinoma raro cuyos hallazgos invasivos son predominantemente en forma de es- 
tructuras papilares y la misma estructura es hallada en las metástasis; se reconocen frecuentes focos de crecimiento papilar intraductal». Los carcinomas papilares que se derivan y limitan a un quiste mamario son considerados como carcinomas intraquísticos no invasivos.

En general constituyen el 1 al $2 \%$ de los carcinomas mamarios y son más comunes en mujeres de mayor edad que el promedio para los demás tumores malignos, con promedios que van desde los 63 hasta los 67 anos. Se presenta descarga por el pezón en el 22 al 34\% de las pacientes. Sereportan metástasis axilares entre el 25 y $32 \%$ y el índice de recurrencias es del $13 \%$.

\section{Carcinoma Mamario Metaplásico}

En un pequeño número de adenocarcinomas el epitelio canceroso asume una apariencia no glandular por un proceso de metaplasia, el cual también puede originarse en tejido benigno como ductos o quistes y en tumores benignos como fibroadenomas y papilomas.

Se describen de dos tipos; escamoso (homólogo) y pseudosarcomatoso (heterólogo). En general los primeros tienen pronóstico mas favorable. La sobrevida a 5 años en el tipo pseudosarcomatoso en estado I es del $44 \%$.

\section{Carcinoma Apocrino}

Aún no se ha definido el significado de la metaplasia apocrina en el carcinoma mamario aunque algunos autores han considerado la transición de hiperplasia con metaplasia apocrina a carcinoma. Sin embargo, el carcinoma apocrino intraductal es una variante histológica in situ diferente del carcinoma ductal que puede existir sola o combinada con el carcinoma apocrino invasivo. Algunos autores lo denominaban como el «sweat gland carcinoma of the breast» (carcinoma sudoríparo de la mama).

La presentación in situ parece tener el mismo comportamiento que los demás carcinomas intraductales, y el pronóstico en las formas infiltrantes no difiere de los demás carcinomas ductales invasivos.

\section{Carcinoma Adenoide Quístico}

Constituye menos del $0,1 \%$, también denominado como Cilindroma.Llama la atención por su favorable pronóstico y apariencia histológica similar a los tumores originados en glandulas salivales.

Se ha reportado buena sobrevida con cirugía y se han descrito metástasis axilares en pocos casos. Se sabe de recurrencias después de 20 años.

\section{Carcinoma Escamoso}

Es una forma extremadamente rara y para ser considerada como tal debe estar totalmente constituída por carcinoma escamoso. Debe ser distinguida de los carcinomas metaplásicos en los cuales el adenocarcinoma es el origen de las células escamosas. Su histogénesis permanece sinexplicación. Algunos casos pueden representar una variante extrema del carcinoma metaplásico.El promedio de edad es de 57 años y el $50 \%$ de las pacientes son mayores de 60 años. El cuadro clínico no es diferente al de los ductales infiltrantes. El tratamiento y el pronóstico tampoco parecen ser distintos.

\section{Tumor Filodes}

El tumor filoides es un tumor generalmente de pronóstico bueno aunque impredecible. Con características histológicas y clínicas parecidas al fibroadenoma; el diagnóstico del tumor filoides es totalmente patológico basado en su componente estromal y su componente epitelial.

Es una lesión rara y representa el $0.5 \%$ de todos los tumores mamarios y con una mayor frecuencia a los 40 años, pero que se puede presentar a cualquier edad.

Son tumores que con frecuencia alcanzan un gran tamaño con un diámetro promedio de 6 a $8 \mathrm{cms}$, son nodulares, indoloros y móviles, no retraen la piel y pueden ulcerarse solo cuando alcanzan grandes volúmenes debido a la isquemia de la misma.

Presentan metástasis de un 3 a un $12 \%$, cifras que aumentan hasta un 40 a $50 \%$ con el tumor filodes maligno. Se han tratado de relacionar los rasgos histológicos con la evolución clínica, clasificando los tumores como histológicamente benignos, malignos y fronterizos. Esta clasificación no ha logrado resolver el problema pronóstico ya que en ocasiones tumores con histología benigna dar metástasis o los tumores que semejan un sarcoma franco no las desarrollan.

El pronóstico es generalmente bueno, aunque impredecible y se presentan recidivas entre un 15 y un $60 \%$ según diferentes autores.

El tratamiento es la resección amplia con margen de $3 \mathrm{cms}$ y seguimiento adecuado.

\section{Carcinoma Secretor}

Este tipo de tumor, también denominado carcinoma juvenil por ocurrir relativamente más frecuente pero no exclusivamente en ninas, en edades en que el carcinoma mamario es extremadamente raro. La primera descripción data de 1966 por Mc Diver en una serie de 7 pacientes entre 3 y 15 años. También han sido descritos casos en edades avanzadas.

La resección local amplia es el tratamiento preferido en ninas, y la mastectomía está indicada en adultas cuando los márgenes son positivos, especialmente en grandes tumores. Las metástasis axilares no ocurren en más del $20 \%$ y de ellas el $50 \%$ se presentan en pacientes de más de 50 anos. El pronóstico en general es favorable.

\section{Carcinoma Mamario con Hallazgos Endocrinos}

Algunos carcinomas mamarios tienen la capacidad de producir hormonas ectópicas y se ha considerado la ocurrencia de una metaplasia endocrina o bioquímica con producción de $\mathrm{HCG}$, calcitonina, epinefrina, $\mathrm{ACTH}$ y paratohormona, detectables por análisis bioquímico, morfológico e inmunohistoquímico.

Un ejemplo lo constituye el carcinoma mamario con diferenciación coriocarcinomatosa, el cual tiene un curso agresivo. 
Se han reportado células aisladas de carcinomas reactivos por inmunohistoquímica a alfa-beta-HCG en un 15 a $20 \%$ de carcinomas ductales infiltrantes corrientes, sin embargo la presencia de estas células no parece tener significado pronóstico y no se han descrito efectos funcionales.

Una forma más frecuente es el carcinoma que contiene gránulos citoplasmáticos argirófilos, hallados entre un 3 a $25 \%$. Las pacientes con carcinomas mucinosos argirófilos presentan más metástasis axilares (48\%) que aquellos tumores con Grimelius negativo (26\%).

Hasta ahora la producción ectópica de hormonas o la diferenciación endocrina no se han considerado como elementos que influyan críticamente en el pronóstico o el tratamiento.

\section{Tumores Metastásicos a la Mama}

Los tumores metastásicos a la mama son muy raros; los más frecuentes son las leucemias y linfomas, el melanoma y el carcinoma de pulmón en especial el de células pequenas; menos comunmente se observan metástasis de tumores de ovario, sarcomas de tejidos blandos, adenocarcinomas gastrointestinales y tumores genitourinarios.

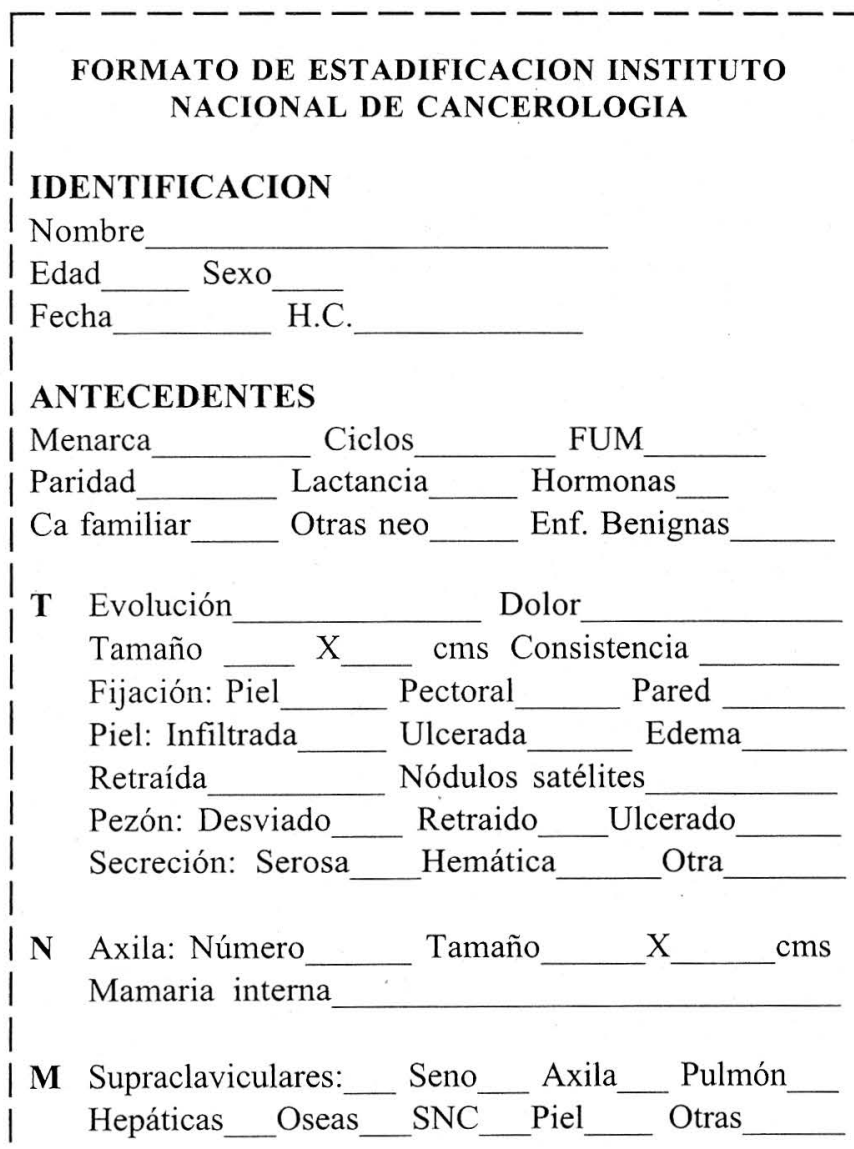

Fecha de biopsia Protocolo No

Diagnóstico Histológico

Receptores Hormonales: Estradiol Progesterona

Marcadores tumorales
La Mamografía muestra hallazgos variables que incluyen masas únicas o múltiples, engrosamiento de la piel o adenopatías axilares; raramente se ven calcificaciones.

El Bacaf es útil para hacer diagnóstico diferencial.

El manejo debe enfocarse de acuerdo con el tumor primario y el pronóstico es malo, la mayoría de las pacientes han fallecido 1 año después del diagnóstico.

\section{Estadificación del Cáncer Mamario por el T.N.M.}

El propósito del sistema TNM es estandarizar la información obtenida de tal forma que la estadificación sea lo más precisa posible desde el punto de vista de la valoración clínica. $\mathbf{T}$ se refiere al tumor, $\mathbf{N}$ al estado de los ganglios linfáticos y $\mathbf{M}$ a la presencia de metástasis.

\section{DEFINICIONES}

\section{TUMOR PRIMARIO ( T )}

TX Tumor primario no puede ser evaluado

T0 No evidencia de tumor Primario

Tis Carcinoma in situ: Carcinoma Intraductal, Carcinoma lobulillar in situ, Enfermedad de Paget del pezón sin tumor.

T1 Tumor de $2 \mathrm{cms}$ o menos en su mayor dimensión

T1a Tumor de $0.5 \mathrm{cms}$ o menos en su mayor dimensión

T1b Tumor mayor de $0.5 \mathrm{cms}$ pero no mayor de $1.0 \mathrm{~cm}$ en su mayor dimensión

T1c Tumor mayor de $1.0 \mathrm{~cm}$ pero no mayor de $2.0 \mathrm{cms}$ en su mayor dimensión

T2 Tumor mayor de $2 \mathrm{cms}$ pero no mayor de $5 \mathrm{cms}$ en su mayor dimensión

T3 Tumor mayor de $5 \mathrm{cms}$ en su mayor dimensión

T4 Tumor de cualquier tamaño con extensión directa a piel o pared torácica

T4a Extensión a pared torácica $\left({ }^{*}\right)$

T4b Edema (incluyendo piel de naranja) o ulceración de la piel de la mama o nódulos cutáneos satélites confinados a la misma mama

T4c Ambos T4a y T4b

T4d Carcinoma inflamatorio

\section{GANGLIOS LINFATICOS (N)}

NX Ganglios linfáticos regionales no pueden ser evaluados

N0 No ganglios linfáticos regionales metastáticos

N1 Metástasis a ganglios linfáticos axilares ipsilaterales móviles

N2 Metástasis a ganglios linfáticos axilares ipsilaterales fijos entre sí o a otras estructuras

N3 Metástasis a ganglios linfáticos mamarios internos ipsilaterales

(*) La pared torácica incluye las costillas, los músculos intercostales y músculo serrato mayor, pero no el músculo pectoral. 


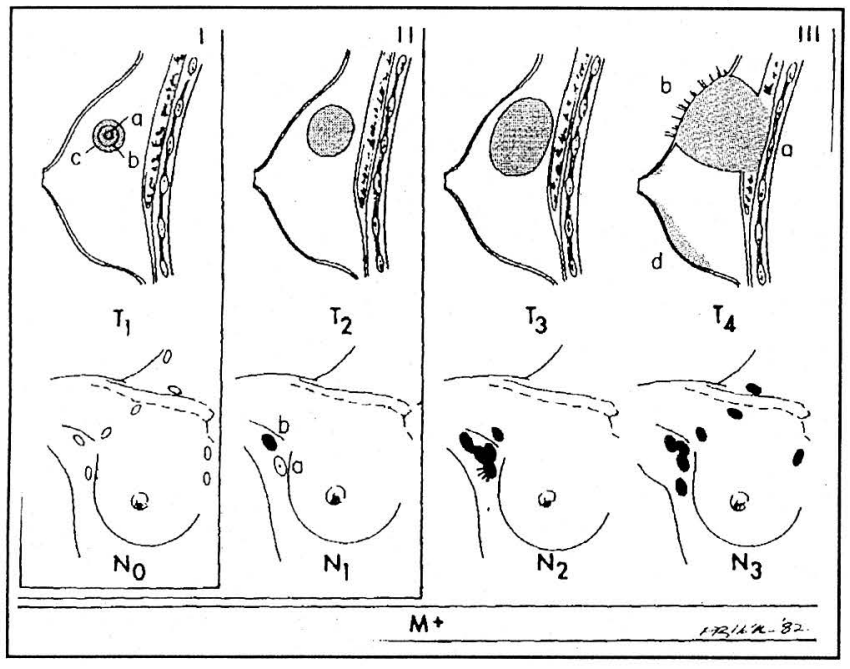

\section{METASTASIS A DISTANCIA (M)}

MX Presencia de metástasis a distancia no puede ser valorada

M0 No hay metástasis a distancia

M1 Metástasis a distancia (incluyendo metástasis a ganglio supraclavicular ipsilateral)

\section{AGRUPAMIENTO POR ESTADOS}

\begin{tabular}{lccc}
\hline ESTADO 0 & Tis & N0 & Mo \\
ESTADO I & T1 & N0 & M0 \\
ESTADO IIA & T0 & N1 & M0 \\
& T1 & N1 & Mo \\
ESTADO IIB & T2 & N0 & M0 \\
& T2 & N1 & M0 \\
ESTADO IIIA & T3 & N0 & M0 \\
& T0 & N2 & M0 \\
& T1 & N2 & M0 \\
& T2 & N2 & M0 \\
& T3 & N1 & M0 \\
ESTADO IIIB & T3 & N2 & M0 \\
& Cualquier T & Cualquier N & M0 \\
ESTADO IV & Cualquier T & Cualquier N & M0 \\
& & & \\
\hline
\end{tabular}

\section{TIPOS HISTOLOGICOS}

\section{CANCER sin otra especificación}

DUCTAL

Intraductal (in situ)

Invasor con componente intraductal
Comedociano

Inflamatorio

Medular con infiltrado linfocítico

Mucinoso (Coloidal)

Papilar

Escirroso

Tubular

Otros

\section{LOBULILLAR}

In situ

Invasor con componente in situ Invasor

DEL PEZON

Enfermedad de Paget sin otra especificación

Enfermedad de Paget con carcinoma intraductal

Enfermedad de Paget con carcinoma ductal invasor

\section{GRADO HISTOPATOLOGICO}

$\begin{array}{ll}\text { GX } & \text { Grado no puede ser valorado } \\ \text { G1 } & \text { Bien diferenciado } \\ \text { G2 } & \text { Moderadamente bien diferenciado } \\ \text { G3 } & \text { Mal diferenciado } \\ \text { G4 } & \text { Indiferenciado }\end{array}$

\section{DEFINICION DEL GRADO DE DIFERENCIACION HISTOLOGICA POR LA ESCALA DE SCARFF- BLOOM-RICHARDSON.}

Este esquema de graduación evalúa estos tres paramétros:

1. Grado de formación tubular.

ptos

$75 \%$ o más del tumor está compuesto por túbulos. 1

10 a $75 \%$ del tumor está compuesto por túbulos. 2

Menos del 10\% del tumor está compuesto por túbulos. 3

\section{Grado nuclear.}

Núcleos pequeños y uniformes

Núcleos moderadamente variables en tamaño y forma

Núcleos con marcado incremento en el tamaño e

irregularidad

\section{Tasa mitótica.}

Dependiente del área y del campo seleccionado.

Objetivo de 40X mitosis por 10 campos es:

0 a 5 mitosis por 10 campos

6 a 10 mitosis por 10 campos

mayor de 11 mitosis por 10 campos

El grado histológico está determinado por la suma de los puntos:

Grado $1=3$ a 5 puntos - bien diferenciado

Grado $2=6$ a 7 puntos - moderadamente diferenciado

Grado 3 = 8 a 9 puntos - pobremente diferenciado

Factores pronóstico 